\title{
Rosmarinus officinalis L. increases Caenorhabditis elegans stress resistance and longevity in a DAF-16, HSF-1 and SKN-1-dependent manner
}

\author{
D.C. Zamberlan ${ }^{1}$, G.P. Amaral ${ }^{1}$, L.P. Arantes ${ }^{1}$, M.L. Machado ${ }^{1}$, C.R. Mizdal ${ }^{2}$, \\ M.M.A. Campos ${ }^{2}$ and F.A.A. Soares ${ }^{1}$ \\ ${ }^{1}$ Centro de Ciências Naturais e Exatas, Departamento de Bioquímica e Biologia Molecular, Programa de Pós-graduação em \\ Ciências Biológicas: Bioquímica Toxicológica, Universidade Federal de Santa Maria, Santa Maria, RS, Brasil \\ ${ }^{2}$ Departamento de Análises Clínicas Toxicológicas, Centro de Ciências da Saúde, \\ Universidade Federal de Santa Maria, Santa Maria, RS, Brasil
}

\begin{abstract}
Improving overall health and quality of life, preventing diseases and increasing life expectancy are key concerns in the field of public health. The search for antioxidants that can inhibit oxidative damage in cells has received a lot of attention. Rosmarinus officinalis L. represents an exceptionally rich source of bioactive compounds with pharmacological properties. In the present study, we explored the effects of the ethanolic extract of $R$. officinalis (eeRo) on stress resistance and longevity using the nonparasitic nematode Caenorhabditis elegans as a model. We report for the first time that eeRo increased resistance against oxidative and thermal stress and extended $C$. elegans longevity in an insulin/IGF signaling pathway-dependent manner. These data emphasize the eeRo beneficial effects on C. elegans under stress.
\end{abstract}

Key words: Caenorhabditis elegans; Natural compounds; Rosemary; daf-2; Stress resistance; Aging

\section{Introduction}

Aging and lifespan of multicellular organisms are affected by several genetic factors. Signal transduction pathways that regulate gene expression in response to extracellular cues are common targets in the search for longevity genes (1). Insulin/IGF signaling is a conserved signal transduction pathway that regulates growth and anabolic functions of multicellular organisms at the expense of cellular stress defenses and repair by modulating stress resistance and longevity (2).

Rosmarinus officinalis L. (Labiatae), popularly known as rosemary, is a common household plant grown in many parts of the world. Aqueous and ethanolic extracts of $R$. officinalis have been shown to contain many substances with pharmacological properties. Health benefits include the following characteristics: antioxidant, antidiabetic (3), hepatoprotective (4), antithrombotic (5), antinociceptive (6), anti-inflammatory (7), antidepressant (8), and gastroprotective (9). Given that $R$. officinalis appears to have beneficial effects in diseases that are strongly linked to aging, we investigated the effects of the ethanolic extract of this plant (eeRo) on aging using the non-parasitic nematode Caenorhabditis elegans as a model.
C. elegans has been shown to be a valuable model in understanding the molecular mechanisms that modulate aging and stress responses. Its short lifespan, fully mapped genome and its application in genetic manipulations have enabled researchers to study the function, regulation and output of insulin/IGF-1 signaling (10). This pathway is highly conserved between worms and mammals. Daf-2 encodes the only insulin/IGF-1 receptor expressed in $C$. elegans. Studies have demonstrated that mutations in daf-2 increase $C$. elegans resistance to oxidative stress (11) and heat stress (12) and lead to an extended lifespan in a DAF16/FOXO-dependent manner (13). Tullet et al. (14) suggested that the transcription factor SKiNhead (SKN-1/Nrf2) directly integrates insulin/IGF signaling and the stress response. Recent studies have also implicated heat shock factor (HSF) as a regulator of longevity that interacts with the insulin pathway $(15,16)$. MEV-1 is also involved in aging and sensitivity to oxidative stress. The expression patterns of the antioxidant enzymes genes superoxide dismutase (sod) and catalase (ctl) mirrored one another in the two mutants daf-16 and mev-1. In addition, both strains were extremely sensitive

Correspondence: F.A.A. Soares: <felix@ufsm.br>

Received February 12, 2016 | Accepted May 6, 2016 
to paraquat, a superoxide anion generator. However, the short life span and oxidative stress-hypersensitivity of daf-16 mutant may result from suppression of anti-oxidant genes, such as sod-1 or sod-3, rather than increase of ROS production from mitochondria as in mev-1 (17).

Studies have proposed that integration of cytoprotective and stress-responsive signaling pathways is crucial for environmental adaptation and hence, control of longevity (18). Despite documentation of the many protective properties of the $R$. officinalis extract, there have been no studies on the signaling pathways that may be involved. C. elegans has the potential to bridge the gap between in vitro and in vivo approaches. This model complements genetic studies and helps in the search for a mechanism of action of the extract. In this study, we investigated the effect of eeRo on $C$. elegans stress resistance and longevity and evaluated the signaling pathways involved.

\section{Material and Methods}

\section{Chemicals and reagents}

Ethanol, 5-hydroxy-1,4-naphthoquinone (juglone) and 5-(and-6)-chloromethyl-2', $7^{\prime}$-dichlorodihydrofluorescein diacetate $\left(\mathrm{CM}-\mathrm{H}_{2} \mathrm{DCFDA}\right)$ were purchased from SigmaAldrich (USA).

The eeRo was obtained from the dried leaves $\left(40^{\circ} \mathrm{C}\right)$ of this plant, which were collected in the botanical garden of Universidade Federal de Santa Maria, Brazil. The leaves were subjected to an alcoholic extraction (100\% ethanol, $1.5 \mathrm{~h}, 60-70^{\circ} \mathrm{C}$ ) in the Soxhlet apparatus with some modification in relation to the original technique (19). High performance liquid chromatography (HPLC-DAD) was previously performed and revealed the presence of the rosmarinic acid, carnosic acid, chlorogenic acid, caffeic acid, quercetin, rutin and kaempferol (12).

\section{C. elegans strains, maintenance and treatment}

The wild-type C. elegans strain N2 (Bristol) and mutant worms TK22 [mev-1(kn1)]; CB1370 [daf-2 (e1370) III]; CF1038 [daf-16(mu86)], PS3551 [hsf-1(sy441)]; EU-1 [skn-1(zu67) IV/nT1] and TJ356 [daf-16p::daf-16a/b:: GFP + rol-6] were obtained from the $C$. elegans Genetics Center (University of Minnesota, Minneapolis, MN, USA).

For all worms, age-synchronized eggs were obtained by isolating embryos from gravid hermaphrodites using bleaching solution ( $1 \% \mathrm{NaOCl}, 0.25 \mathrm{M} \mathrm{NaOH})$. For EU-1 worms, we grew synchronized animals and plated young adults. skn-1 worms are uncoordinated (Unc), while skn-1 homozygotes are non-Unc, allowing us to enrich for skn-1 homozygotes using a plate crawling assay. The L1 population was transferred to $10 \mathrm{~mL} \mathrm{NGM} \mathrm{(nematode}$ growth medium) plates seeded with Escherichia. coli OP50 as a food source and eeRo at 10,25 or $50 \mu \mathrm{g} / \mathrm{mL}$ or vehicle $(0.1 \% \mathrm{EtOH})$ and allowed to develop. The strain CB1370 was maintained at $16^{\circ} \mathrm{C}$ since it is sensitive to dauer formation at $20^{\circ} \mathrm{C}$. The wild-type and the other mutant strains were maintained at $20^{\circ} \mathrm{C}$.

\section{Bacterial growth assay}

The minimum inhibitory concentration (MIC) in E. coli OP50 was performed according to Clinical and Laboratory Standards Institute with few modifications (20). Bacteria was seeded onto plates with Mueller Hinton agar and allowed to grow for $24 \mathrm{~h}$ at $37^{\circ} \mathrm{C}$. We then prepared suspensions of microorganisms in Mueller Hinton broth. Fifty microliters of the standardized microorganism suspension was placed in each well of a 96-well microliter plate, along with an equal volume of compound to be tested at different concentrations. We performed broth, growth, and compound vehicle controls to which the results were compared. The plates were incubated for $24 \mathrm{~h}$ at $37^{\circ} \mathrm{C}$. The MIC was considered as the lowest concentration of the test product able to inhibit the growth of microorganisms evidenced by the use of $2,3,5$ triphenyltetrazolium chloride $1 \%$.

\section{Oxidative stress resistance}

Synchronized L1-larva N2 and mutant strains were transferred to treatment plates containing eeRo or vehicle (control) and allowed to develop at $20^{\circ} \mathrm{C}$ up to adulthood (approximately 2 days). The pretreated worms were collected, washed three times with M9 buffer and transferred into Eppendorf tubes. A volume of $10 \mu \mathrm{L}$ of $10 \mathrm{mM}$ juglone (stock solution freshly prepared) was added to the micro tubes containing 1000 worms in $990 \mu \mathrm{L}$ of M9. The worms were exposed to $100 \mu \mathrm{M}$ juglone (final concentration) during $1 \mathrm{~h}$, washed three times with M9 buffer and transferred to NGM plates containing vehicle or eeRo. After $24 \mathrm{~h}$, the number of living worms was counted and reported as percent of the control. The mutant assays were conducted using the concentration that was deemed most effective in increasing survival (in wild type).

\section{Thermotolerance assay}

Synchronized 1-day-adult N2 and mutant worms, pretreated with eeRo or vehicle (control) since L1-larval stage, were exposed to $35^{\circ} \mathrm{C}$ for $4 \mathrm{~h}$. After this procedure the plates were returned to $20^{\circ} \mathrm{C}$ for 24 additional hours. The number of survivors were scored. The mutant assays were conducted using the concentration that was deemed most effective in increasing survival (in wild type).

\section{Lifespan assay}

The lifespan assay of $C$. elegans was investigated as previously described (21). The pre-fertile period of adulthood was used as time zero $(\mathrm{t}=0)$. The worms were kept on NGM plates containing eeRo or vehicle (control) and $E$. coli just in the middle of the plate and transferred to new plates every two days. Nematodes were regarded as dead if they did not move after repeated stimulus. They were excluded if they crawled away from the plate. 
The maximum lifespan was defined as the $10 \%$ of last survival population. The mutant assays were conducted using the concentration that was deemed most effective in inducing lifespan extension (in wild type). Blinding of studies was not possible due to the color of eeRo, which stains the NGM agar. Experiments were performed at least in triplicate with 100 nematodes each.

\section{Quantification of ROS}

Intra-worm ROS generation was measured in C. elegans wild-type and mutant strains using $\mathrm{CM}-\mathrm{H}_{2}$ DCFDA, following a previously described method (22) with minor modifications. Briefly, L1 age-synchronized worms were transferred onto culture plates containing either vehicle or eeRo. The worms were maintained at $20^{\circ} \mathrm{C}$ until adulthood $(\sim 48 \mathrm{~h})$. After, they were collected, washed with fresh M9 buffer three times and transferred to micro tubes. The worms were exposed to $25 \mathrm{mM} \mathrm{H}_{2} \mathrm{O}_{2}$ (final concentration, induced groups) or vehicle (basal groups) during $1 \mathrm{~h}$ and washed again with $\mathrm{M} 9$ buffer. After, $10 \mu \mathrm{L}$ of $2 \mathrm{mM} \mathrm{CM}-\mathrm{H}_{2}$ DCFDA were added to the Eppendorf tubes containing 1000 worms in $990 \mu \mathrm{L}$ M9 (20 $\mu \mathrm{M} \mathrm{CM}-\mathrm{H}_{2}$ DCFDA final concentration) and incubated for $2 \mathrm{~h}$. The mutant worms mev-1 were just exposed to $\mathrm{CM}-\mathrm{H}_{2}$ DCFDA. The worms were washed and transferred to 96-well plates (100 worms per well). The fluorescence intensity was measured with a plate reader (Excitation: $488 \mathrm{~nm}$; Emission: $510 \mathrm{~nm}$ ). The mutant assays were conducted using the concentration that was deemed most effective in decreasing ROS levels (in wild type).

\section{DAF-16 localization}

For each slide, a minimum of 20 worms were mounted on $4 \%$ agarose pads in $\mathrm{M} 9$ and anaesthetized with $10 \mathrm{mM}$ sodium azide. Fluorescence was acquired with an epifluorescence microscope housed in air-conditioned rooms $\left(20^{\circ} \mathrm{C}\right)$.

\section{Statistical analyses}

Statistical analyses were performed using GraphPad (Prism for Windows, Version 5.01 GraphPad Software, USA). Significance was assessed by one-way analysis of variance (ANOVA), followed by Newman-Keuls test, or two-way ANOVA, followed by Bonferroni's test for post hoc comparison. Significance for survival analysis was assessed by the Kaplan-Meier curve followed by the logrank test for trend. Values of $\mathrm{P}<0.05$ were considered to be statistically significant.

\section{Results}

\section{Effect of eeRo on resistance to oxidative stress}

To test the antioxidant effect of eeRo in vivo, we monitored $C$. elegans survival under oxidative stress. Exposure to $100 \mu \mathrm{M}$ juglone for $1 \mathrm{~h}$ induced an approximately $50 \%$ mortality rate in wild-type worms (Figure $1 \mathrm{~A}$ ). Treatment with 10 and $100 \mu \mathrm{g} / \mathrm{mL}$ eeRo did not have any
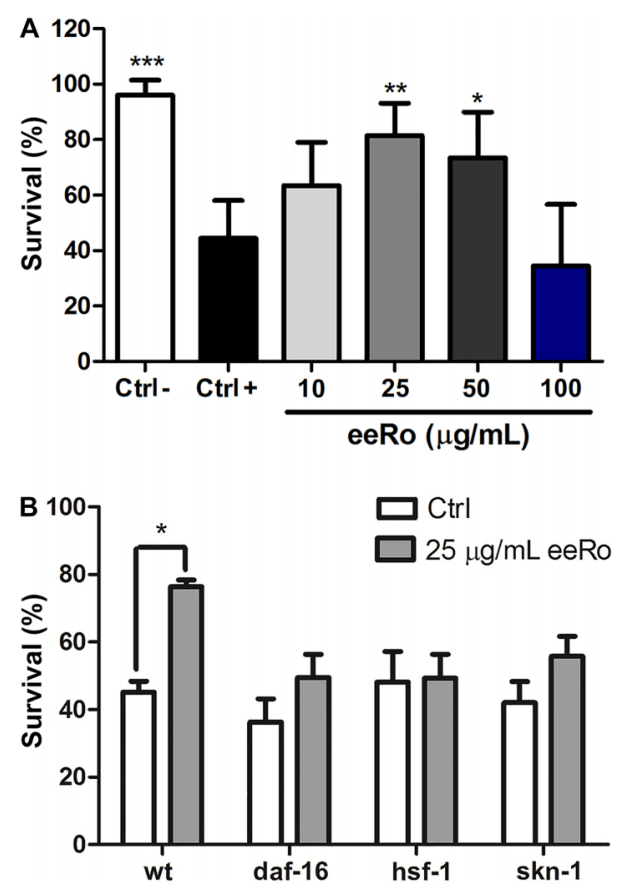

Figure 1. Effect of ethanolic extract of Rosmarinus officinalis $L$. (eeRo) on juglone-induced mortality. Survival of wild-type $(A)$ and mutant worms $(B)$ treated with eeRo and exposed to $100 \mu \mathrm{M}$ juglone for $1 \mathrm{~h}(\mathrm{Ctrl}+)$. Data are reported as percentage of living worms of 100 worms per group in each experiment from 4 independent assays. $A,{ }^{*} \mathrm{P}<0.05$, ${ }^{* *} \mathrm{P}<0.01$, ${ }^{* * *} \mathrm{P}<0.001$, compared to the Ctrl + group (one-way ANOVA). $B$, *P $<0.05$ (two-way ANOVA).

effect on survival rate. In contrast, worms treated with 25 and $50 \mu \mathrm{g} / \mathrm{mL}$ eeRo had a significantly lower mortality rate compared to the untreated induced control group (Figure 1A, Ctrl+). The most effective eeRo concentration in decreasing mortality was $25 \mu \mathrm{g} / \mathrm{mL} \quad(P<0.01)$. We investigated the effect of this dose on the survival rate against juglone exposure in daf-16, hsf-1 and skn-1 mutants. We found no significant differences in mortality among the strains evaluated. Furthermore, treatment with $25 \mu \mathrm{g} / \mathrm{mL}$ eeRo did not decrease mortality rate in the mutants, as observed in wild-type worms (Figure 1B).

\section{Effect of eeRo on thermal tolerance}

Worms were subjected to thermal stress at $35^{\circ} \mathrm{C}$ for $4 \mathrm{~h}$, which induced a mortality of approximately $50 \%$ in wild-type worms (Figure 2A). Treatment with 25 and $50 \mu \mathrm{g} / \mathrm{mL}$ eeRo significantly decreased mortality in 28 and $24 \%$, respectively, in wild-type worms compared to the control group. The most effective concentration of eeRo was $25 \mu \mathrm{g} / \mathrm{mL}(P<0.01)$. We performed the thermal tolerance assay with daf-16, $h s f-1$ and skn-1 mutants. No significant differences in thermal resistance were observed among the untreated strains, although increased mortality was observed in daf-16. No significant differences in 

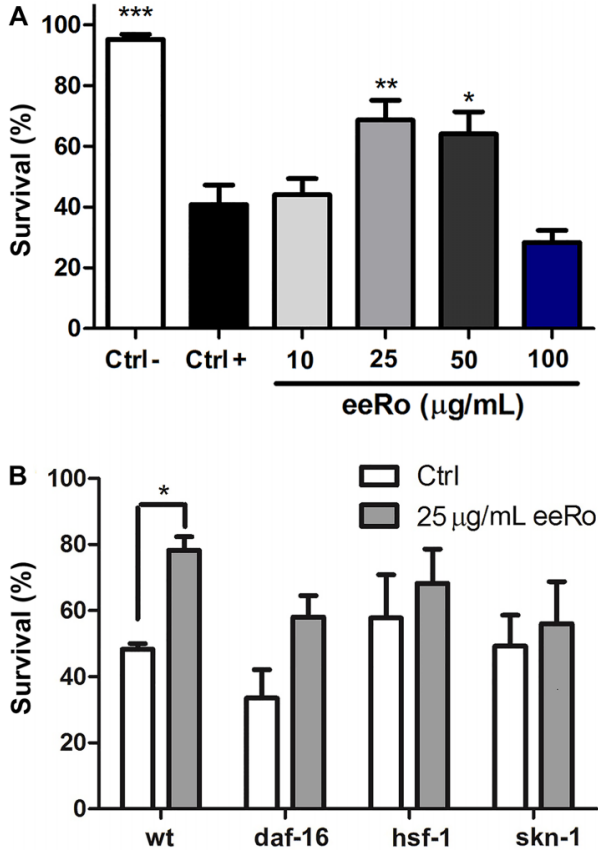

Figure 2. Effect of ethanolic extract of Rosmarinus officinalis $L$. (eeRo) on thermal stress. Survival of wild-type $(A)$ and mutant worms $(B)$ treated with eeRo and exposed to thermal stress $\left(35^{\circ} \mathrm{C}\right)$ for $4 \mathrm{~h}(\mathrm{Ctrl}+)$. Data are reported as percentage of living worms of 100 worms in each experiment from 4 independent assays. $A,{ }^{*} \mathrm{P}<0.05,{ }^{* *} \mathrm{P}<0.01,{ }^{* * *} \mathrm{P}<0.001$, compared to the $\mathrm{Ctrl}+$ group (one-way ANOVA). $B,{ }^{*} \mathrm{P}<0.05$ (two-way ANOVA).

mortality were noted in daf-16, hsf-1 and skn-1 mutants treated with $25 \mu \mathrm{g} / \mathrm{mL}$ eeRo compared to the untreated group (Figure 2B).

\section{Effect of eeRo on ROS production}

We used the wild-type strain to investigate if eeRo decreased basal ROS production and ROS induced by $\mathrm{H}_{2} \mathrm{O}_{2}$ exposure. There was a significant increase in ROS levels induced by $1 \mathrm{~h}$ exposure to $25 \mathrm{mM} \mathrm{H}_{2} \mathrm{O}_{2}$ (Figure 3A). Worms treated with eeRo at 10, 25, and $50 \mu \mathrm{g} / \mathrm{mL}$ had significantly lower basal ROS production than untreated worms. Moreover, eeRo treatment also prevented an increase in ROS levels induced by $\mathrm{H}_{2} \mathrm{O}_{2}$ in the wild-type strain compared to the untreated induced control group $(P<0.05)$.

We quantified ROS levels in TK22 (mev-1) mutant. Figure 3B shows significantly higher ROS levels in the transgenic worms compared to the wild-type strain $(P<0.001)$. At all concentrations tested, the worms treated with eeRo had significantly lower ROS levels compared to the untreated worms $(P<0.001)$. This decrease in ROS levels was most evident at an eeRo concentration of $25 \mu \mathrm{g} / \mathrm{mL}$.

Measurement of ROS production in daf-16, hsf-1 and skn-1 mutants demonstrated that they have higher levels of reactive species than the wild type (Figure $3 C$ ). Treatment with $25 \mu \mathrm{g} / \mathrm{mL}$ eeRo significantly decreased ROS levels in these mutants compared to untreated worms $(P<0.01)$. However, the effects of eeRo in the mutants were less apparent than in the wild-type strain $(\mathrm{P}<0.001)$

\section{Effect of eeRo on DAF-16 translocation}

No differences in DAF-16 translocation were observed in worms treated with eeRo compared to untreated worms (data not shown).

\section{Effect of eeRo on lifespan}

To test the effects of eeRo on C. elegans longevity we monitored time-course survival in wild type and mutant worms. The mean survival time was extended by treatment with $25 \mu \mathrm{g} / \mathrm{mL}$ eeRo in the wild-type strain from 12- (control) to 15-day adults (treated). Similarly, an increase in maximum lifespan also was observed from 16- (control) to 20-day adults (treated) (Table 1). A possible explanation for the beneficial effects of eeRo on aging in $C$. elegans is that these compounds increased cellular stress resistance. To confirm this, we performed a lifespan assay in TK22 (mev-1) mutant. mev-1 had a decreased lifespan compared to the wild type strain. EeRo treatment extended the mev-1 mean survival-time from 10 to 12 days at a concentration of $25 \mu \mathrm{g} / \mathrm{mL}$ (Table 1). The maximum lifespan of mev-1 was extended from 16 (control) to 18 days following treatment with $25 \mu \mathrm{g} / \mathrm{mL}$ eeRo (Figure 4B; Table 1).

We performed the lifespan assay in daf-2, daf-16, hsf-1 and skn-1 mutants with and without $25 \mu \mathrm{g} / \mathrm{mL}$ eeRo treatment (Figure 5). EeRo treatment increased the mean lifespan of daf-16 from 10- (control) to 12-day adults (treated), but not the maximum lifespan. In daf-2 mutant, we observed a slight increase in the maximum lifespan from 30 to 32 days in worms treated with the extract. EeRo treatment did not have an effect on lifespan in the hsf-1 and skn-1 mutants, which had mean survival times of 8 and 9 days, respectively, and maximum lifespans of 13 and 14 days, respectively (Table 2 ).

\section{Effects of eeRo on E. coli OP50}

To verify whether eeRo influenced $E$. coli OP50 growth, we assessed the minimum inhibitory concentration (MIC). EeRo did not have a significant antimicrobial effect on $E$. coli OP50 growth at a concentration range between $0.39-50 \mu \mathrm{g} / \mathrm{mL}$ (data not shown).

\section{Discussion}

The search for antioxidants from natural sources has received a lot of attention. Antioxidants can inhibit cellular oxidative damage and prevent development of related diseases. The plant $R$. officinalis represents an exceptionally rich source of different bioactive compounds (23). 

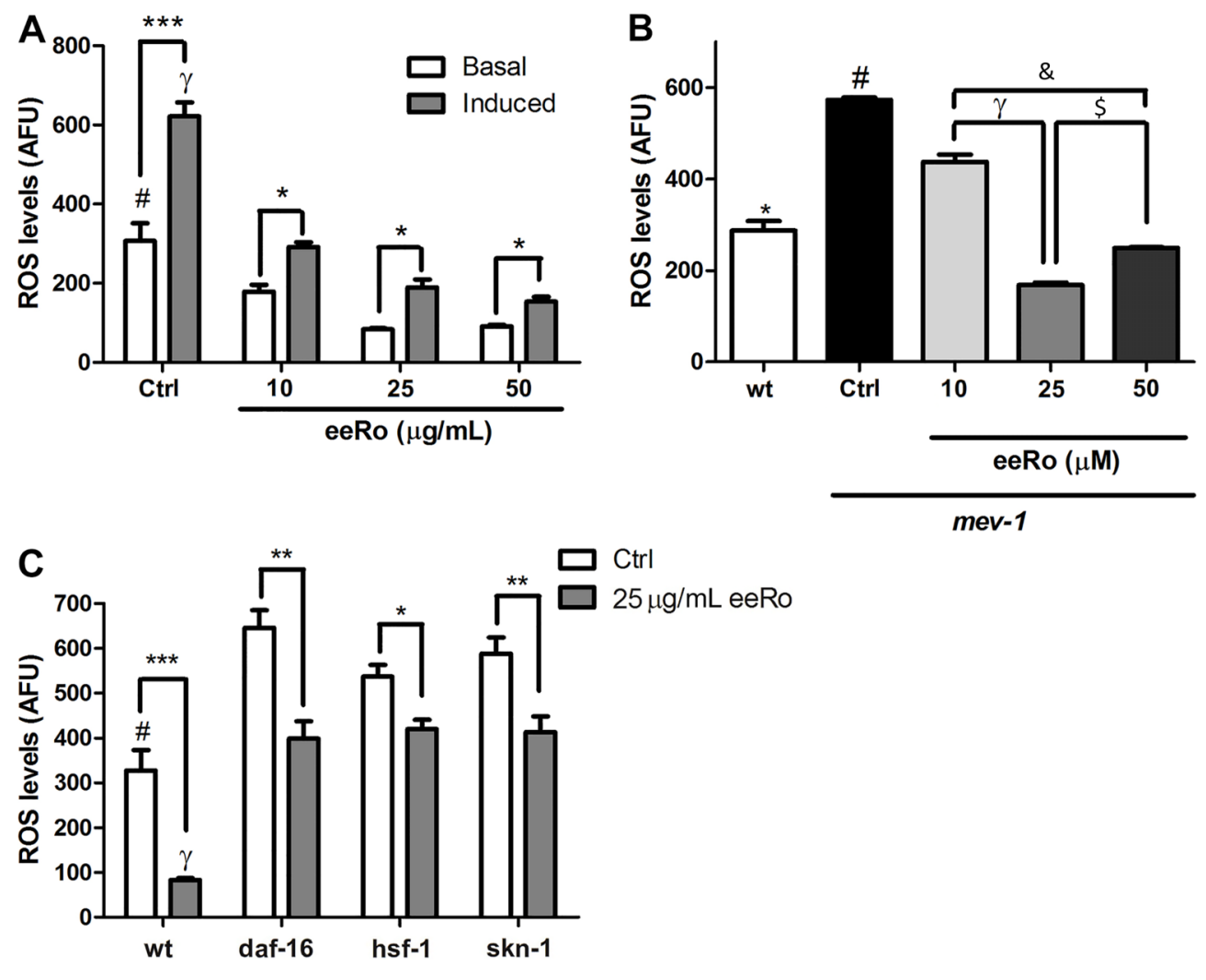

Figure 3. Effect of ethanolic extract of Rosmarinus officinalis $L$. (eeRo) on reactive oxygen species (ROS) production. Data are reported in arbitrary fluorescence units (AFU) from 3 independent assays $(n=3)$. A, Levels of basal and $\mathrm{H}_{2} \mathrm{O}_{2}$-induced $(25 \mathrm{mM} / 1 \mathrm{~h}) \mathrm{ROS}$ production in wild-type (N2) worms. ${ }^{*} \mathrm{P}<0.001$ compared to eeRo-treated basal groups; ${ }^{\#} \mathrm{P}<0.001$ compared to eeRo-treated induced groups; ${ }^{\gamma} \mathrm{P}<0.01 \mathrm{Ctrl}$ basal compared to Ctrl induced groups. $B$, Levels of ROS production in wild-type (N2) and mev-1 (TK22) worms. ${ }^{*} \mathrm{P}<0.001$, compared to all other groups; ${ }^{\#} \mathrm{P}<0.001$, compared to all other groups; ${ }^{\gamma} \mathrm{P}<0.001,10 \mu \mathrm{M}$ compared to the $25 \mu \mathrm{M}$ eeRo treated group; ${ }^{\$} \mathrm{P}<0.001,25 \mu \mathrm{M}$ compared to the $50 \mu \mathrm{M}$ eeRo treated group; ${ }^{\&} \mathrm{P}<0.001,10 \mu \mathrm{M}$ compared to the $50 \mu \mathrm{M}$ eeRo treated group (one-way ANOVA). C, ROS levels in daf-16, hsf-1 and skn-1 mutants treated or not with the extract. ${ }^{*} P<0.05$, ${ }^{* *} P<0.01$, ${ }^{* * *} \mathrm{P}<0.001$, treated compared to untreated group; ${ }^{\#} \mathrm{P}<0.01$, compared to the other Ctrl groups; ${ }^{\gamma} \mathrm{P}<0.01$, compared to the other treated groups (two-way ANOVA).

Table 1. Lifespan of wild-type worms treated with eeRo $(\mu \mathrm{g} / \mathrm{mL})$.

\begin{tabular}{lcc}
\hline & Median lifespan (days) & Maximum lifespan (days) \\
\hline Control & $12 \pm 1.15$ & $16 \pm 0.57$ \\
10 & $12 \pm 1.00$ & $17 \pm 1.00$ \\
25 & $15 \pm 1.15^{\star}$ & $20 \pm 1.15^{\star}$ \\
50 & $13 \pm 1.73$ & $17 \pm 1.52$ \\
\hline
\end{tabular}

Data are reported as means $\pm \mathrm{SD}$. Aging assays were performed at $20^{\circ} \mathrm{C}$ except for daf-2, which was carried out at $16^{\circ} \mathrm{C}$. Experiments were repeated three times starting with 90 nematodes per group. ${ }^{*} \mathrm{P}<0.05$, significantly different from untreated control group by the log-rank (Mantel-Cox) test.

The eeRo used in this study includes flavonoids (quercetin, rutin and kaempferol) and phenolic acids (chlorogenic, caffeic, rosmarinic and carnosic acids) as the most abundant components (9). R. officinalis appears to have beneficial effects in prevalent diseases that are strongly linked to aging, such as diabetes and cancer. In the present study, we explored the possibility that this plant can influence aging using the non-parasitic nematode C. elegans as a model. We report for the first time that the eeRo increased worm resistance against oxidative and thermal stress and extended $C$. elegans longevity in an insulin/IGF signaling-dependent manner.

Juglone is a naphthoquinone that induces superoxide anion radicals overproduction (24). Juglone can cause premature death at concentrations that overload the organism's protective capacity (24). As we previously described, $1 \mathrm{~h}$ of $100 \mu \mathrm{M}$ juglone-exposition induces a lethality of approximately $50 \%$ of wild-type worms (25). The present study demonstrated that the eeRo treatment was able to decrease the juglone-induced mortality by reducing $\mathrm{ROS}$ production and protecting against oxidative 

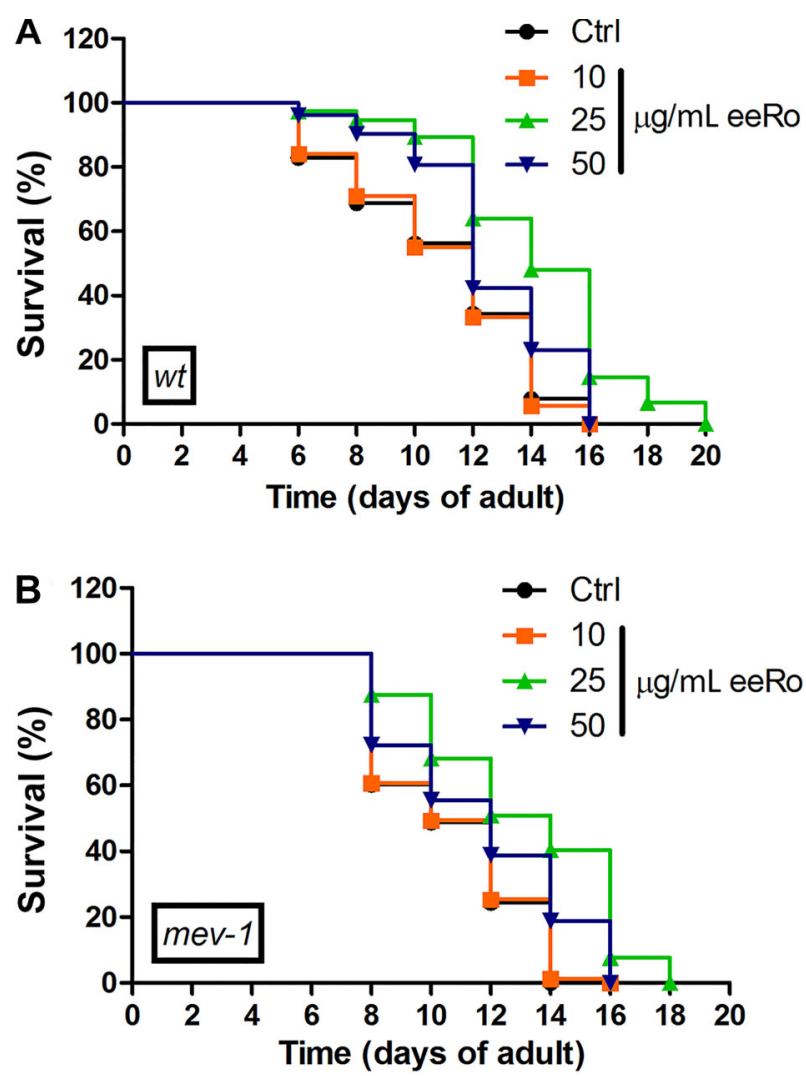

Figure 4. Effect of ethanolic extract of Rosmarinus officinalis $L$. (eeRo) on $C$. elegans lifespan. Survival curves were significantly extended in $(A)$ wild-type and $(B)$ mev-1 worms treated with $25 \mu \mathrm{g} / \mathrm{mL}$ eeRo $(\mathrm{P}<0.0001$, log-rank (Mantel-Cox) test)

damage. These data corroborate the extract antioxidant properties previously mentioned (3). In addition, thermal tolerance was significantly increased by eeRo treatment in wild-type worms (Figure 2A). Previous studies demonstrated that thermal stress causes an increase of ROS levels in the worms and therefore it is likely that the death of worms was at least partially due to oxidative stress (26). These outcomes are known to depend partly on intracellular stress signaling pathways that are activated in response to oxidative stress and as a consequence of direct damage to DNA, proteins, and lipids. These cellular injuries and signaling mechanisms modulate transcription factor activities resulting in changes to gene expression profiles (27). In this way, given that both thermal and chemical stresses result in similar changes in gene expression, eeRo may modulate signaling pathways that are crucial to defense processes. These findings emphasize the potential of eeRo against environmental stress.

Active insulin/IGF signaling promotes phosphorylationdependent cytoplasmic sequestration of the transcription factors DAF-16/FOXO, HSF-1 and SKN-1/Nrf2 $(14,28)$. Under favorable environmental conditions, signaling through this pathway activates a conserved PI3-kinase/AKT cascade, which causes phosphorylation of DAF-16/FOXO, thereby allowing reproductive development (29). The transcription factor HSF-1 guides DAF-16/FOXO activity and cooperatively induces transcription of a subset of target genes, including heat shock proteins involved in proteostasis (1). SKN-1/Nrf regulates resistance to oxidative stress and expression of detoxification genes (2). We performed survival assays in daf-16, hsf-1 and skn-1 mutants and found no significant differences among treated and untreated worms. Moreover, we observed that these strains were more sensitive to oxidative and thermal stress compared to the wild type. These data indicate that DAF-16, HSP-1 and SKN-1 expression, involved in the insulin/IGF signaling network, and the activation of target genes, are essential for eeRo to exert its effect. However, the extract decreased ROS levels in the transgenic strains tested, although less efficiently than in the wild-type. This data suggests that the constituents of the extract may be acting as direct scavengers and then reducing ROS production in the mutants. Besides, more studies are necessary in order to evaluate whether the eeRo capacity to decrease ROS production occurs by modulating the antioxidant system.

In several studies, increased longevity has been closely associated with improved survival under conditions of heat or oxidative stress. In accordance with those studies, eeRo treatment extended mean and maximum lifespan of wild-type worms, likely by increasing cellular stress resistance. Many common stress-induced effects on physiology, gene expression and signaling pathways among animals have been reported (30).

Studies have also demonstrated single gene mutations that influence lifespan. mev-1 encodes the $C$. elegans ortholog of the succinate dehydrogenase cytochrome $b$ subunit, which is required for oxidative phosphorylation. Mutations in mev-1 result in premature aging and increased sensitivity to oxidative stress (31). Attention has also focused on the insulin-like signaling pathway in C. elegans because of its pivotal role in lifespan determination and oxidative stress resistance $(32,33)$. DAF-16 is a well-known regulator of longevity (32). Studies have also implicated heat shock factor (HSF) as an influence on longevity (15). Tullet et al. (14) suggested that SKN-1/Nrf2 directly integrates insulin/IGF signaling and the stress response.

We performed survival assays using mev-1, daf-2, daf-16, hsf-1 and skn-1 transgenic strains. Activity of the DAF-2 insulin/IGF receptor regulates both L1 arrest and dauer formation in C. elegans. Complete loss of daf-2 function leads to $L 1$ arrest and lethality at $20^{\circ} \mathrm{C}$ (34). Due to this, we performed the daf-2 mutant lifespan assay at $16^{\circ} \mathrm{C}$. Because the eeRo concentration at $25 \mu \mathrm{g} / \mathrm{mL}$ was most efficient at decreasing the mortality rate and protecting the worms, we performed survival assays with mutants using this dose. We tested daf-2 mutant in order 

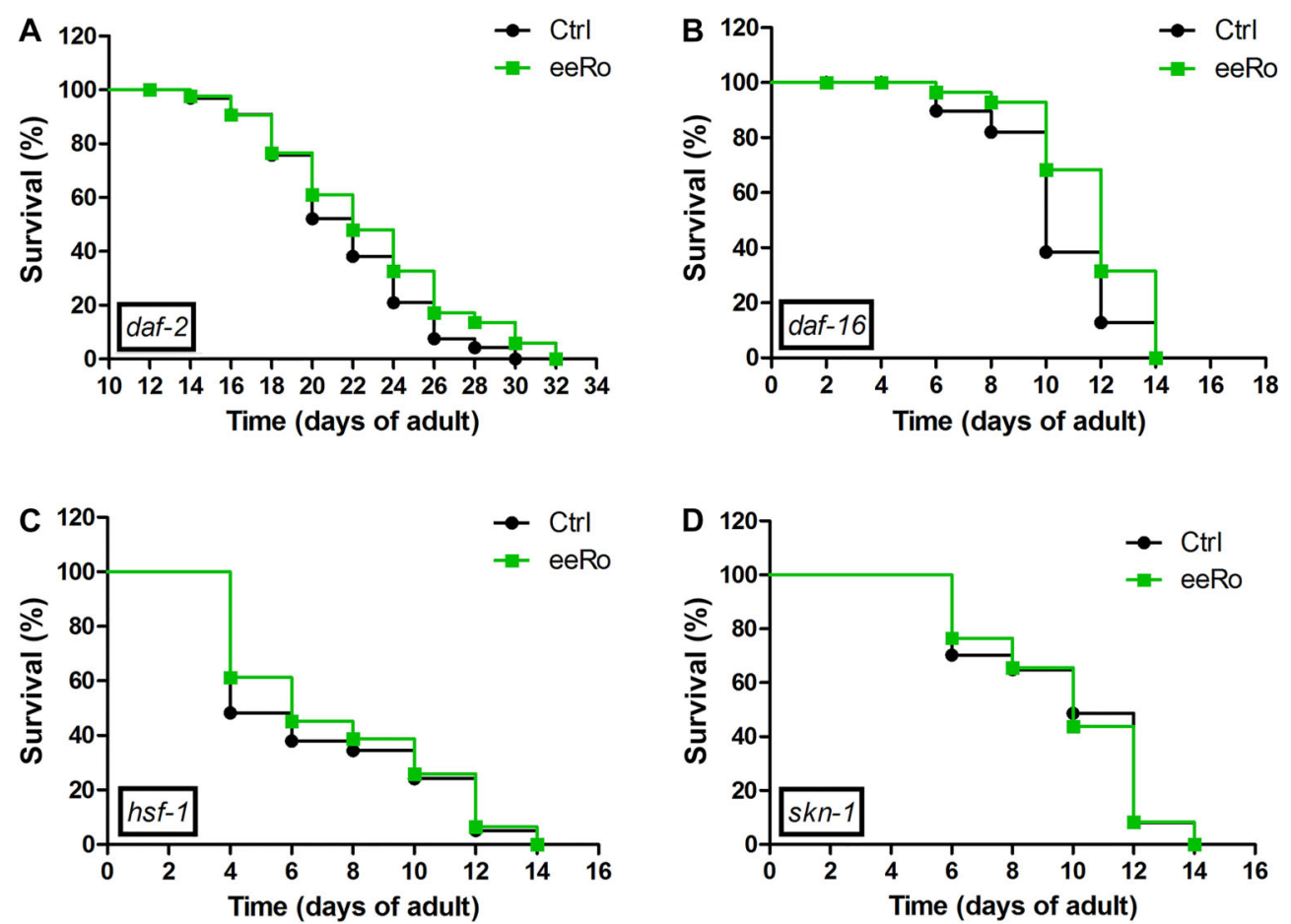

Figure 5. Effect of $25 \mu \mathrm{g} / \mathrm{mL}$ ethanolic extract of Rosmarinus officinalis L. (eeRo) in $(A)$ daf-2, $(B)$ daf-16, $(C)$ hsf-1 and $(D)$ skn-1 mutants lifespans. Survival curves were significantly different in daf-16 (median lifespan) and in daf-2 (maximum lifespan) $(P<0.05$, log-rank (Mantel-Cox) test).

Table 2. Lifespan of mutant worms treated with $25 \mu \mathrm{g} / \mathrm{mL}$ eeRo.

\begin{tabular}{|c|c|c|c|c|}
\hline \multirow[t]{2}{*}{ Genotype } & \multicolumn{2}{|c|}{$\begin{array}{c}\text { Median } \\
\text { lifespan (days) }\end{array}$} & \multicolumn{2}{|c|}{$\begin{array}{c}\text { Maximum } \\
\text { lifespan (days) }\end{array}$} \\
\hline & Control & eeRo & Control & eeRo \\
\hline mev-1 & $10 \pm 1.00$ & $12 \pm 0.57^{*}$ & $16 \pm 0.57$ & $18 \pm 1.15^{*}$ \\
\hline$d a f-2$ & $22 \pm 1.75$ & $22 \pm 0.57$ & $30 \pm 1.15$ & $32 \pm 1.52^{*}$ \\
\hline daf-16 & $10 \pm 0.57$ & $12 \pm 1.73^{*}$ & $14 \pm 2.00$ & $15 \pm 1.15$ \\
\hline$h s f-1$ & $8 \pm 1.15$ & $8 \pm 2.00$ & $13 \pm 1.15$ & $14 \pm 0.57$ \\
\hline skn-1 & $9 \pm 0.86$ & $10 \pm 1.75$ & $14 \pm 0.57$ & $14 \pm 1.15$ \\
\hline
\end{tabular}

Data are reported as means $\pm \mathrm{SD}$. Aging assays were performed at $20^{\circ} \mathrm{C}$ except for daf-2, which was carried out at $16^{\circ} \mathrm{C}$. Experiments were repeated three times starting with 90 nematodes per group. ${ }^{*} \mathrm{P}<0.05$, significantly different from untreated control group by the log-rank (Mantel-Cox) test.

to investigate if the eeRo effect on longevity was just through this pathway. In daf-2 and daf-16 survival curves, there was a slight difference between treated and untreated worms, in a less pronounced way compared to wild type. These data suggest a partial dependence of DAF-2 and DAF-16. These findings suggest that eeRo and daf-2 mutants may extend adult lifespan through overlapping mechanisms that are not additive. Furthermore, our data demonstrated that DAF-16, $\mathrm{HSF}-1$ and $\mathrm{SKN}-1$ are required for the extract to exert its protective effect given that the treatment did not significantly decrease juglone- or thermal stress-induced mortality in daf-16, hsf-1 and skn-1 mutants, as observed in wild-type worms. Furthermore, the treatment did not extend $h s f-1$ and skn-1 lifespan, as observed in the wild type worms, emphasizing the need of these target genes for eeRo protective effect.

DAF-16 is crucial for many important processes, including development, stress resistance, thermal tolerance 
and metabolism (35-38). Along with hsf-1, daf-16 is part of the heat-shock response in C. elegans (28). The HSF plays essential and evolutionarily conserved roles in the activation of heat shock-inducible gene expression. HSFs are recognized as regulators of stress-induced gene expression, besides contributing to more complex organismal physiological processes such as development, growth, aging, immunity, and reproduction. We suggest that the beneficial effects of eeRo on aging in $C$. elegans are based on increased cellular stress resistance in a manner partially dependent on IIS pathway activation of target genes. We demonstrated that eeRo modulated the cellular response to oxidative stress in mev-1 mutants, decreasing ROS levels and extending the lifespan. Previous studies have shown that treatment with natural compounds, such as Ginkgo biloba extract, increased mev-1 mutant resistance to acute oxidative and thermal stress (39). Mutations in daf-16 and mev-1 resulted in similar patterns of hypersensitivity, with several interesting differences. The short lifespan and oxidative stress hypersensitivity of the daf-16 mutant resulted from suppressed anti-oxidant gene expression rather than an increase in ROS production from the mitochondria, as in mev-1. In mev-1 mutant, DAF-16 is present in the nuclei even under normal conditions (40). This observation leads to the prediction that eeRo affects both nematode ROS production and anti-oxidant gene expression.

This study demonstrates the potential protective effect of eeRo in C. elegans, as evidenced by an increase in tolerance against oxidative and thermal stress, a decrease in ROS production and extension of longevity in HSF1- and SKN-1-dependent interactions. These findings suggest that eeRo triggers the signaling pathways that lead to

\section{References}

1. Guarente L, Kenyon C. Genetic pathways that regulate ageing in model organisms. Nature 2000; 408: 255-262, doi: $10.1038 / 35041700$.

2. Kuningas M, Mooijaart SP, van Heemst D, Zwaan BJ, Slagboom PE, Westendorp RG. Genes encoding longevity: from model organisms to humans. Aging Cell 2008; 7: 270-280, doi: 10.1111/j.1474-9726.2008.00366.x.

3. Bakirel T, Bakirel U, Keles OU, Ulgen SG, Yardibi H. In vivo assessment of antidiabetic and antioxidant activities of rosemary (Rosmarinus officinalis) in alloxan-diabetic rabbits. J Ethnopharmacol 2008; 116: 64-73, doi: 10.1016/j.jep. 2007.10.039.

4. Sotelo-Felix JI, Martinez-Fong D, Muriel P, Santillan RL, Castillo D, Yahuaca P. Evaluation of the effectiveness of Rosmarinus officinalis (Lamiaceae) in the alleviation of carbon tetrachloride-induced acute hepatotoxicity in the rat. J Ethnopharmacol 2002; 81: 145-154, doi: 10.1016/ S0378-8741(02)00090-9.

5. Yamamoto J, Yamada K, Naemura A, Yamashita T, Arai R. Testing various herbs for antithrombotic effect. Nutrition 2005; 21: 580-587, doi: 10.1016/j.nut.2004.09.016. transcriptional activation of downstream targets, which are essential for the effects described in C. elegans. These transcription factors play key roles in insulin/IGF-1 signaling, and several additional pathway components have been shown to modulate aging in flies, mice and possibly humans, implying that the effects of the pathway on aging and stress resistance are conserved.

We report for the first time that eeRo increased the resistance against oxidative and thermal stress and extended $C$. elegans longevity in a DAF-16, HSF-1 and SKN-1-dependent manner. These survival-enhancing effects of eeRo on $C$. elegans at both normal conditions and under stress emphasize the potential of $R$. officinalis to promote resistance against oxidative damage in these worms through the activation of related genes. As the death rate of a population is closely related to external stresses, it could be concluded that the survival-enhancing effects of eeRo on $C$. elegans under stress are very important for antiaging research.

\section{Acknowledgments}

The authors are thankful to the Caenorhabditis elegans Genetics Center (University of Minnesota, Minneapolis, MN, USA). The authors are also thankful to Instituto Nacional de Ciência e Tecnologia (INCT) for Excitotoxicity and Neuroprotection - MCT/CNPq, Programa de Apoio a Núcleos Emergentes (PRONEM/FAPERGS; \#11/2029-1), and CNPq Universal (\#472669/2011-7 and \#475896/2012-2) for providing financial assistance. F.A.A. Soares, L.P. Arantes, G.P. Amaral, and M.M.A. de Campos received fellowships from $\mathrm{CNPq}$ and D.C. Zamberlan and M.L. Machado received fellowships from CAPES.
6. Gonzalez-Trujano ME, Pena El, Martinez AL, Moreno J, Guevara-Fefer P, Deciga-Campos M, et al. Evaluation of the antinociceptive effect of Rosmarinus officinalis L. using three different experimental models in rodents. J Ethnopharmacol 2007; 111: 476-482, doi: 10.1016/ j.jep.2006.12.011.

7. Altinier G, Sosa S, Aquino RP, Mencherini T, Della LR, Tubaro A. Characterization of topical antiinflammatory compounds in Rosmarinus officinalis L. J Agric Food Chem 2007; 55: 1718-1723, doi: 10.1021/jf062610+.

8. Heinrich M, Kufer J, Leonti M, Pardo-de-Santayana M. Ethnobotany and ethnopharmacology - interdisciplinary links with the historical sciences. J Ethnopharmacol 2006; 107: 157-160, doi: 10.1016/j.jep.2006.05.035.

9. Amaral GP, de Carvalho NR, Barcelos RP, Dobrachinski F, Portella RL, da Silva MH, et al. Protective action of ethanolic extract of Rosmarinus officinalis L. in gastric ulcer prevention induced by ethanol in rats. Food Chem Toxicol 2013; 55: 48-55, doi: 10.1016/j.fct.2012.12.038.

10. Brenner S. The genetics of Caenorhabditis elegans. Genetics 1974; 77: 71-94. 
11. Honda $\mathrm{Y}$, Honda $\mathrm{S}$. The daf-2 gene network for longevity regulates oxidative stress resistance and Mn-superoxide dismutase gene expression in Caenorhabditis elegans. FASEB J 1999; 13: 1385-1393.

12. Lithgow GJ, White TM, Melov S, Johnson TE. Thermotolerance and extended life-span conferred by single-gene mutations and induced by thermal stress. Proc Natl Acad Sci U S A 1995; 92: 7540-7544, doi: 10.1073/pnas.92.16.7540.

13. Kenyon C, Chang J, Gensch E, Rudner A, Tabtiang R. A C. elegans mutant that lives twice as long as wild type. Nature 1993; 366: 461-464, doi: 10.1038/366461a0.

14. Tullet JM, Hertweck M, An JH, Baker J, Hwang JY, Liu S, et al. Direct inhibition of the longevity-promoting factor SKN-1 by insulin-like signaling in C. elegans. Cell 2008; 132: 1025-1038, doi: 10.1016/j.cell.2008.01.030.

15. Morley JF, Morimoto RI. Regulation of longevity in Caenorhabditis elegans by heat shock factor and molecular chaperones. Mol Biol Cell 2004; 15: 657-664, doi: 10.1091/ mbc.E03-07-0532.

16. Steinkraus KA, Smith ED, Davis C, Carr D, Pendergrass WR, Sutphin GL, et al. Dietary restriction suppresses proteotoxicity and enhances longevity by an hsf-1-dependent mechanism in Caenorhabditis elegans. Aging Cell 2008; 7: 394-404, doi: 10.1111/j.1474-9726.2008.00385.x.

17. Yanase S, Yasuda K, Ishii N. Adaptive responses to oxidative damage in three mutants of Caenorhabditis elegans (age-1, mev-1 and daf-16) that affect life span. Mech Ageing Dev 2002; 123: 1579-1587, doi: 10.1016/ S0047-6374(02)00093-3.

18. Baumeister R, Schaffitzel E, Hertweck M. Endocrine signaling in Caenorhabditis elegans controls stress response and longevity. J Endocrinol 2006; 190: 191-202, doi: 10.1677/joe.1.06856.

19. Ashok P, Koti BC, Thippeswamy AH, Tikare VP, Dabadi $P$, Viswanathaswamy $\mathrm{AH}$. Evaluation of antiinflammatory activity of Centratherum anthelminticum (L) Kuntze seed. Indian J Pharm Sci 2010; 72: 697-703, doi: 10.4103/0250474X.84577.

20. Wayne. Methods for dilution antimicrobialsusceptibility tests for bacteria that grow aerobically: approved standard. CLSI document M7-A7 \%7 7th \%! Methods for dilutio; 2006.

21. Schlotterer A, Kukudov G, Bozorgmehr F, Hutter H, Du X, Oikonomou D, et al. C. elegans as model for the study of high glucose-mediated life span reduction. Diabetes 2009; 58: 2450-2456, doi: 10.2337/db09-0567.

22. Sakaue Y, Kim J, Miyamoto Y. Effects of TAT-conjugated platinum nanoparticles on lifespan of mitochondrial electron transport complex I-deficient Caenorhabditis elegans, nuo-1. Int $J$ Nanomedicine 2010; 5: 687-695, doi: 10.2217/ nnm.10.47.

23. Borras-Linares I, Stojanovic Z, Quirantes-Pine R, ArraezRoman D, Svarc-Gajic J, Fernandez-Gutierrez A, et al. Rosmarinus officinalis leaves as a natural source of bioactive compounds. Int J Mol Sci 2014; 15: 20585-20606, doi: 10.3390/ijms151120585.

24. Blum J, Fridovich I. Superoxide, hydrogen peroxide, and oxygen toxicity in two free-living nematode species. Arch Biochem Biophys 1983; 222: 35-43, doi: 10.1016/00039861(83)90499-X.

25. Zamberlan DC, Arantes LP, Machado ML, Golombieski R, Soares FA. Diphenyl-diselenide suppresses amyloid-beta peptide in Caenorhabditis elegans model of Alzheimer's disease. Neuroscience 2014; 278: 40-50, doi: 10.1016/ j.neuroscience.2014.07.068.

26. Michalski Al, Johnson TE, Cypser JR, Yashin Al. Heating stress patterns in Caenorhabditis elegans longevity and survivorship. Biogerontology 2001; 2: 35-44, doi: 10.1023/ A:1010091315368.

27. Johnson TE, Wood WB. Genetic analysis of life-span in Caenorhabditis elegans. Proc Natl Acad Sci U S A 1982; 79: 6603-6607, doi: 10.1073/pnas.79.21.6603.

28. Hsu AL, Murphy CT, Kenyon C. Regulation of aging and agerelated disease by DAF-16 and heat-shock factor. Science 2003; 300: 1142-1145, doi: 10.1126/science.1083701.

29. Dorman JB, Albinder B, Shroyer T, Kenyon C. The age-1 and daf-2 genes function in a common pathway to control the lifespan of Caenorhabditis elegans. Genetics 1995; 141: 1399-1406.

30. Lant B, Storey KB. An overview of stress response and hypometabolic strategies in Caenorhabditis elegans: conserved and contrasting signals with the mammalian system. Int J Biol Sci 2010; 6: 9-50, doi: 10.7150/ijbs.6.9.

31. Ishii N, Fujii M, Hartman PS, Tsuda M, Yasuda K, SenooMatsuda $\mathrm{N}$, et al. A mutation in succinate dehydrogenase cytochrome b causes oxidative stress and ageing in nematodes. Nature 1998; 394: 694-697, doi: 10.1038/29331.

32. Lin K, Hsin H, Libina N, Kenyon C. Regulation of the Caenorhabditis elegans longevity protein DAF-16 by insulin/ IGF-1 and germline signaling. Nat Genet 2001; 28: 139-145, doi: $10.1038 / 88850$.

33. Lee SS, Kennedy S, Tolonen AC, Ruvkun G. DAF-16 target genes that control $C$. elegans life-span and metabolism. Science 2003; 300: 644-647, doi: 10.1126/science.1083614.

34. Gems D, Sutton AJ, Sundermeyer ML, Albert PS, King KV, Edgley ML, et al. Two pleiotropic classes of daf-2 mutation affect larval arrest, adult behavior, reproduction and longevity in Caenorhabditis elegans. Genetics 1998; 150: 129-155.

35. Vowels JJ, Thomas JH. Genetic analysis of chemosensory control of dauer formation in Caenorhabditis elegans. Genetics 1992; 130: 105-123.

36. Murakami S, Johnson TE. A genetic pathway conferring life extension and resistance to UV stress in Caenorhabditis elegans. Genetics 1996; 143: 1207-1218.

37. Henderson ST, Johnson TE. daf-16 integrates developmental and environmental inputs to mediate aging in the nematode Caenorhabditis elegans. Curr Biol 2001; 11: 1975-1980, doi: 10.1016/S0960-9822(01)00594-2.

38. Ogg S, Paradis S, Gottlieb S, Patterson GI, Lee L, Tissenbaum $\mathrm{HA}$, et al. The Fork head transcription factor DAF-16 transduces insulin-like metabolic and longevity signals in C. elegans. Nature 1997; 389: 994-999, doi: 10.1038/40194.

39. Wu Z, Smith JV, Paramasivam V, Butko P, Khan I, Cypser JR, et al. Ginkgo biloba extract EGb 761 increases stress resistance and extends life span of Caenorhabditis elegans. Cell Mol Biol 2002; 48: 725-731.

40. Kondo M, Senoo-Matsuda N, Yanase S, Ishii T, Hartman PS, Ishii N. Effect of oxidative stress on translocation of DAF-16 in oxygen-sensitive mutants, mev-1 and gas-1 of Caenorhabditis elegans. Mech Ageing Dev 2005; 126: 637-641, doi: 10.1016/j.mad.2004.11.011. 